\title{
Neural differences between the processing of musical meaning conveyed by direction of pitch change and natural music in congenital amusia
}

Article

Accepted Version

Creative Commons: Attribution-Noncommercial-No Derivative Works 4.0

Zhou, L., Liu, F., Jing, X. and Jiang, C. (2017) Neural differences between the processing of musical meaning conveyed by direction of pitch change and natural music in congenital amusia. Neuropsychologia, 96. pp. 29-38. ISSN 0028-3932 doi:

https://doi.org/10.1016/j.neuropsychologia.2016.12.024 Available at https://centaur.reading.ac.uk/68567/

It is advisable to refer to the publisher's version if you intend to cite from the work. See Guidance on citing.

Published version at: http://www.sciencedirect.com/science/article/pii/S0028393216304638

To link to this article DOI:

http://dx.doi.org/10.1016/j.neuropsychologia.2016.12.024

Publisher: Elsevier

All outputs in CentAUR are protected by Intellectual Property Rights law, including copyright law. Copyright and IPR is retained by the creators or other copyright holders. Terms and conditions for use of this material are defined in the End User Agreement. 


\section{www.reading.ac.uk/centaur}

\section{CentAUR}

Central Archive at the University of Reading

Reading's research outputs online 
Neural differences between the processing of musical meaning conveyed by direction of pitch change and natural music in

\title{
congenital amusia
}

\author{
Linshu Zhou ${ }^{\mathrm{a}}$, Fang Liu ${ }^{\mathrm{b}}$, Xiaoyi Jing ${ }^{\mathrm{a}}$, Cunmei Jiang ${ }^{\mathrm{a}^{*}}$ \\ ${ }^{a}$ Music College, Shanghai Normal University, Shanghai, China \\ ${ }^{b}$ School of Psychology and Clinical Language Sciences, University of Reading, Reading, \\ $U K$
}

${ }^{*}$ Corresponding author at: Music College, Shanghai Normal University, 100 Guilin Road, Xuhui District, Shanghai 200234, China.

E-mail addresses: cunmeijiang@126.com (C. Jiang) 


\section{Abstract}

Music is a unique communication system for human beings. Iconic musical meaning is one dimension of musical meaning, which emerges from musical information resembling sounds of objects, qualities of objects, or qualities of abstract concepts. The present study investigated whether congenital amusia, a disorder of musical pitch perception, impacts processing of iconic musical meaning. With a cross-modal semantic priming paradigm, target images were primed by semantically congruent or incongruent musical excerpts, which were characterized by direction (upward or downward) of pitch change (Experiment 1), or were selected from natural music (Experiment 2). Twelve Mandarin-speaking amusics and 12 controls performed a recognition (implicit) and a semantic congruency judgment (explicit) task while their EEG waveforms were recorded. Unlike controls, amusics failed to elicit an N400 effect when musical meaning was represented by direction of pitch change, regardless of the nature of the tasks (implicit versus explicit). However, the N400 effect in response to musical meaning in natural musical excerpts was observed for both groups in both types of tasks. These results indicate that amusics are able to process iconic musical meaning through multiple acoustic cues in natural musical excerpts, but not through direction of pitch change. This is the first study to investigate the processing of musical meaning in congenital amusia, providing evidence in support of the "melodic contour deafness hypothesis" with regard to iconic musical meaning processing in this disorder.

Keywords: Congenital amusia; Iconic musical meaning; Pitch direction; Implicit task; Explicit task; N400 


\section{Introduction}

Congenital amusia (hereafter amusia) is a neurogenetic disorder of pitch processing (Ayotte, Peretz, \& Hyde, 2002; Peretz et al., 2002). Research has suggested that there are two core aspects of musical pitch deficits in amusia. One is the deficit in discriminating fine-grained musical pitch (Foxton, Dean, Gee, Peretz, \& Griffiths, 2004; Hyde \& Peretz, 2004; Jiang, Hamm, Lim, Kirk, \& Yang, 2011), and the other is the deficit in perceiving melodic contour or pitch direction (Foxton et al., 2004; Jiang, Hamm, Lim, Kirk, \& Yang, 2010; Liu, Patel, Fourcin, \& Stewart, 2010). Amusic individuals also show elevated psychophysical thresholds for pitch change detection and pitch direction discrimination and identification (Foxton et al., 2004; Jiang, Lim, Wang, \& Hamm, 2013; Liu et al., 2010; Liu, Xu, Patel, Francart, \& Jiang, 2012; Loui, Guenther, Mathys, \& Schlaug, 2008; Williamson, Liu, Peryer, Grierson, \& Stewart, 2012). The hypothesis of a short-term pitch memory deficit in amusia has been proposed (see Tillmann, Lévêque, Fornoni, Albouy, \& Caclin, 2016 for a review), despite the fact that pitch perception and memory are interwoven in the processing of music (Cook, 1987; Huron \& Parncutt, 1993). Neuroimaging studies suggest that amusia is associated with reduced connectivity between the auditory and inferior frontal cortices in the brain (Albouy et al., 2013; Hyde, Zatorre, \& Peretz, 2011; Loui, Alsop, \& Schlaug, 2009).

Implicit and explicit processing of sound sequences involves distinct neural mechanisms (van Zuijen, Simoens, Paavilainen, Näätänen, \& Tervaniemi, 2006). It has been reported that amusic individuals can perceive harmonic structure implicitly (Tillmann, Gosselin, Bigand, 
\& Peretz, 2012), but cannot perceive tonality and musical syntax in an explicit manner (Jiang, Liu, \& Thompson, 2016). The dissociation between implicit and explicit processing of musical structure has also been shown in other studies (Omigie, Pearce, \& Stewart, 2012; Tillmann, Lalitte, Albouy, Caclin, \& Bigand, 2016; Zendel, Lagrois, Robitaille, \& Peretz, 2015). For example, Zendel et al. (2015) found that an early right anterior negativity (ERAN) in response to melodic deviants was only present in amusics when they attended to non-pitch information (i.e., a click), but not when they attended to pitch (i.e., a note). It may be the case that amusics acquired implicit knowledge of musical structure through passive exposure to music in daily life, despite not being able to show explicit knowledge in laboratory conditions.

Music is an important communicative system for human beings, and deciphering musical meaning is a primary motivation for music listeners. From the perspective of evolution, both primates and humans have the tendency to imitate observed movements for the purpose of communication (Meltzoff \& Prinz, 2002; Nagy, 2006). This is the case in music. Iconic musical meaning, a dimension of musical meaning, emerges from musical information resembling sounds of objects, qualities of objects, or qualities of abstract concepts, while other forms of musical meaning such as intramusical meaning arise from structural relations between musical events, e.g., indexical and symbolic sign quality of music (Koelsch, 2012). In particular, the direction of pitch change (upward or downward) induces iconic musical meaning by imitating an upward or a downward staircase in pitch, while chord sequences ending on a music-syntactically regular or an irregular chord give rise to different 
intramusical meanings. Given amusics' deficits in perceiving pitch change direction (Foxton et al., 2004; Jiang et al., 2013; Liu et al., 2010; Liu, Xu, et al., 2012; Loui et al., 2008; Williamson et al., 2012), it would be worthwhile to examine whether amusic individuals exhibit deficits in the processing of iconic musical meaning conveyed by the direction of pitch change. Exploring this hypothesis would provide evidence for or against the "melodic contour deafness hypothesis" proposed by Patel (2008) for amusia.

The aim of the present study was to examine the presence/absence of an electrophysiological correlate of iconic musical meaning processing in individuals with and without amusia. Given the dissociation between implicit and explicit processing of musical structure in amusia (e.g., Tillmann, Lalitte, et al., 2016; Zendel et al., 2015), we included both implicit and explicit tasks in our experiment. Cross-modal semantic priming has been widely employed in previous investigations of extramusical meaning, in which music excerpts were used to prime semantically congruent/incongruent words (e.g., Koelsch et al., 2004; Painter \& Koelsch, 2011) or images (e.g., Kamiyama, Abla, Iwanaga, \& Okanoya, 2013; Zhou, Jiang, Delogu, \& Yang, 2014). An N400 effect has been observed in both implicit (recognition) and explicit (semantic relatedness judgment) tasks (Koelsch et al., 2004; Zhou, Jiang, Wu, \& Yang, 2015). In the present study, we used the cross-modal semantic priming paradigm to study musical meaning processing, by including musical excerpts and images as primes and targets in both implicit (recognition) and explicit (semantic congruency judgment) tasks. In Experiment 1, we investigated whether the direction of pitch change would prime semantically congruent/incongruent images in amusia. We predicted that the amusic brain 
might respond abnormally to musical meaning representated by direction of pitch change, given their pitch direction deficits. In order to fully understand iconic musical meaning perception in amusia, Experiment 2 examined whether amusic individuals would be able to process iconic musical meaning embedded in natural musical excerpts. We predicted that the amusic brain might respond normally to meaning in natural musical excerpts in which non-pitch based cues (tempo, rhythm, dynamics, and timbre) were available.

\section{Method}

\subsection{Participants}

Participants were recruited through advertisements in the bulletin board systems of universities in Shanghai. Their musical abilities were measured by the Montreal Battery of Evaluation of Amusia (MBEA; Peretz et al., 2003). Twelve amusics and 12 matched controls took part in the experiments. Table 1 shows the participants' information and the mean scores of the MBEA for the two groups. We also calculated the d' of the MBEA for each participant, and the d' for each amusic was below the cutoffs in previous studies (Henry \& McAuley, 2013; Pfeifer \& Hamann, 2015). All participants were native speakers of Mandarin, right-handed, with normal hearing, normal or corrected-to-normal vision, and no history of psychiatric or neurological diseases. None of them had received any extracurricular training in music. Hand dominance was assessed by the Edinburgh Handedness Inventory (Oldfield, 1971). Ethical approval was obtained from Shanghai Normal University, and all participants signed a written consent form before the experiments were conducted. 
Insert Table 1, about here.

\subsection{Electroencephalogram (EEG) recording and analysis}

EEG data were recorded from 64 standard scalp locations (International 10-20 system), digitized at a rate of $500 \mathrm{~Hz}$, with a $0.05 \mathrm{~Hz}$ low cutoff filter and a $100 \mathrm{~Hz}$ high cutoff filter. The data were referenced off-line to the algebraical mean of left and right mastoid electrodes, filtered offline with a band-pass filter of 0.1 to $30 \mathrm{~Hz}$ (24-dB/oct slope). Trials were averaged offline with an epoch length of $900 \mathrm{~ms}$, including a baseline from $100 \mathrm{~ms}$ to $0 \mathrm{~ms}$ before the target onset. Trials with artifacts exceeding the amplitude of $\pm 75 \mu \mathrm{V}$ in any channel were rejected. After statistical evaluation, event-related potentials (ERPs) were, for presentation purposes only, low-pass filtered at $11 \mathrm{~Hz}$ (41 points, FIR).

Based on previous research on the N400 (see Koelsch, 2011 for a review), the time window from 300 to $500 \mathrm{~ms}$ after the onset of target stimulus was selected for statistical analysis. The selected electrodes are shown in Figure 1. Separate repeated measures ANOVAs were conducted for the midline and lateral electrodes. For the midline electrodes, group (amusics, controls) was considered as a between-subjects factor, whereas congruency (congruent, incongruent), task (implicit, explicit), and anteriority (anterior, central, and posterior) were considered as within-subjects factors. For the lateral electrodes, hemisphere (left, right) was added as an additional within-subjects factor. 
Insert Figure 1, about here.

The mean of the respective electrodes in each region of interest was computed for analysis. Only the significant effects containing the main experimental variables (group, congruency, and task) were reported. Overall ANOVAs were followed up by simple effects tests if there were significant two-way interactions between the main experimental variables. All pair-wise comparisons were adjusted by Bonferroni correction. Greenhouse-Geisser correction was applied when the degree of freedom in the numerator was greater than 1 ; in such cases, the original degrees of freedom with corrected $p$ values were reported.

\section{Experiment 1: Musical meaning represented by direction of}

\section{pitch change}

The aim of Experiment 1 was to investigate whether amusics would activate the iconic representation of musical meaning when it was represented by the direction of pitch change in both implicit and explicit tasks. Musical excerpts served as prime stimuli, which were constructed by successive notes with either an upward or a downward direction (see Figure 2 and Supplementary Material for examples). Half of the melodies were composed with small pitch distances no more than 2 semitones, whereas the other half were composed with large pitch distances more than 4 semitones in order to ensure that pitch direction changes were distinguishable to amusics. Real-world scene images depicting an upward or a downward movement served as target stimuli (see Figure 2 for examples). Given that the dissociation 
between implicit and explicit pitch processing in amusia has been found in a previous ERP study (Zendel et al., 2015), it was expected that amusic individuals would elicit an N400 effect in the implicit task, but not in the explicit task.

Insert Figure 2, about here.

\subsection{Stimuli and procedure}

A total of 240 musical excerpts were composed as potential prime stimuli and 240 images were constructed as potential target stimuli. Two pretests were conducted to assess whether the musical excerpts were semantically congruent or incongruent with the images, as well as to ensure that there was no influence of emotional congruency on the music-image pairs. In the end, 160 musical excerpts were selected as the prime stimuli and 160 images were used as the target stimuli, resulting in 320 music-image pairs. The 320 prime-target pairs were assigned to two lists for implicit and explicit tasks, respectively (see Supplementary Material for details).

In the implicit task, the stimuli were split into 20 blocks, each containing 8 stimulus pairs. For each block, participants were instructed to focus on both the musical and pictorial stimuli. After each block, a probe stimulus (either a musical excerpt or an image) was presented, and participants were asked to judge whether the probe stimulus had been presented in the preceding block by pressing one of the two response buttons.

For the explicit task, participants were instructed to focus on the semantic congruency 
between the musical and pictorial stimuli. After the presentation of each prime-target pair, they were required to judge whether the music-image pair was congruent or incongruent by pressing one of the two response buttons.

Consistent with previous studies (e.g., Zendel et al., 2015; Zhou et al., 2015), the implicit task was conducted first so as to prevent participants from using conscious strategies to associate the prime with the target during implicit processing. The entire experiment lasted for approximately $1.5 \mathrm{~h}$, including preparation, instructions, practice, and experimental sessions.

\subsection{Results and discussion}

\subsubsection{Implicit judgment task}

\subsubsection{Behavioral results}

Recognition accuracy of all probe stimuli, visual (images) and auditory (musical excerpts) modalities combined, was calculated. An independent samples $t$ test revealed no significant difference in recognition accuracy between the two groups $\left(t_{(22)}=1.75, p=.09\right.$; amusics: $M=91.25 \%, S D=4.83$; controls: $M=95.00 \%, S D=5.64)$. Furthermore, we also calculated the accuracy of recognition of the musical excerpts that served as probe stimuli, and found no significant difference between the amusics and controls $\left(t_{(22)}=0.30, p=.76\right.$; amusics: $M=95.00 \%, S D=6.74$; controls: $M=95.83 \%, S D=6.69)$. This indicates that both groups attended to and were able to recognize the prime and target stimuli in the recognition (implicit) task. 


\subsubsection{Electrophysiological results}

Figure 3 shows the grand average waveforms elicited by target images following the congruent and incongruent priming of musical excerpts at 9 representative electrodes in the implicit task. The incongruent condition elicited a large negativity with a peak latency of approximately $400 \mathrm{~ms}$ relative to the congruent condition for controls, but not for amusics.

Insert Figure 3, about here.

This was supported by statistical analysis of the standard N400 time window of 300 to $500 \mathrm{~ms}$, which revealed significant three-way interactions among group, congruency, and anteriority, for both lateral $\left(F_{(2,44)}=6.85, p<.05, \varepsilon=.61\right.$, partial $\left.\eta^{2}=.24\right)$ and midline $\left(F_{(2,44)}\right.$ $=6.90, p<.01, \varepsilon=.70$, partial $\eta^{2}=.24$ ) electrodes. Separate two-way ANOVAs were performed for amusics and controls, with congruency and anteriority as within-subjects factors. For amusics, the analysis did not reveal any significant main effect or interaction, for either lateral or midline electrodes $(p s>.26)$. However, for controls, there was a significant interaction between congruency and anteriority, for both lateral $\left(F_{(2,22)}=11.85, p<.01\right.$, $\varepsilon=.59$, partial $\left.\eta^{2}=.52\right)$ and midline $\left(F_{(2,22)}=10.72, p<.01\right.$, partial $\left.\eta^{2}=.49\right)$ electrodes. Further simple effects tests showed that, for controls, the incongruent target elicited a larger N400 than the congruent target over the anterior (lateral: $F_{(1,11)}=30.40, p<.001$, partial $\eta^{2}$ $=.73$; midline: $F_{(1,11)}=21.56, p<.001$, partial $\left.\eta^{2}=.66\right)$ and central (lateral: $F_{(1,11)}=11.11$, $p<.01$, partial $\eta^{2}=.50$; midline: $F_{(1,11)}=8.51, p=.01$, partial $\left.\eta^{2}=.44\right)$ regions, but not over 
the posterior region (lateral: $F_{(1,11)}=1.44, p=.26$, partial $\eta^{2}=.12$; midline: $F_{(1,11)}=1.08, p$ $=.32$, partial $\left.\eta^{2}=.09\right)$.

To further examine whether pitch distance impacts iconic musical meaning processing, ANOVAs taking pitch distance (small versus large) as an additional within-subjects factor were performed. The analysis did not reveal any significant main effect or interaction related to pitch distance, in either lateral or midline electrodes $(p s>.14)$.

\subsubsection{Explicit judgment task}

\subsubsection{Behavioral results}

Accuracy of semantic congruency judgment was calculated. ANOVAs taking group and congruency as factors showed a main effect of group $\left(F_{(1,22)}=43.97, p<.001\right.$, partial $\eta^{2}$ $=.67)$, but no effect of congruency or congruency $\times$ group interaction $(p s>.20)$. Controls outperformed amusics in judging semantic congruency between the primes and targets (amusics: $M=54.42 \%, S D=6.88$; controls: $M=81.33 \%, S D=12.41$ ). However, accuracy rates were comparable between congruent and incongruent conditions, for both amusics [congruent: $56.88 \%(S D=13.73)$; incongruent: $51.77 \%(S D=12.73) ; t_{(11)}=0.78, p=.45$ ] and controls [congruent: $83.75 \%(S D=14.08)$; incongruent: $78.75 \%(S D=14.70) ; t_{(11)}=$ $1.20, p=.26]$.

To further investigate the role of pitch distance, ANOVAs taking pitch distance as an additional within-subjects factor were performed. However, there was no significant main effect or interaction related to pitch distance $(p s>.32)$. 
Correlation analyses revealed that participants' performance on semantic congruency judgment was significantly correlated with their scores on the contour subtest of the MBEA $\left(r_{(22)}=0.66, p<.001\right)$, their melodic scores of the MBEA $\left(r_{(22)}=0.71, p<.001\right)$, and their global MBEA scores $\left(r_{(22)}=0.72, p<.001\right)$. However, these correlations failed to reach significance when only the amusic or control group was considered $(p s>.22)$.

\subsubsection{Electrophysiological results}

Figure 4 shows the grand average waveforms elicited by target images following the congruent and incongruent priming of musical excerpts at 9 representative electrodes in the explicit task. Similar to the implicit task, the incongruent condition elicited a large negativity with a peak latency of approximately $400 \mathrm{~ms}$ relative to the congruent condition for controls, but not for amusics.

Insert Figure 4, about here.

Statistical analysis revealed a significant main effect of congruency (lateral: $F_{(1,22)}=$ $7.67, p<.05$, partial $\eta^{2}=.26$; midline: $F_{(1,22)}=5.07, p<.05$, partial $\left.\eta^{2}=.19\right)$. Moreover, a significant two-way interaction was found between group and congruency, for both lateral ( $F$ $(1,22)=17.09, p<.001, \varepsilon=.61$, partial $\left.\eta^{2}=.44\right)$ and midline $\left(F_{(2,44)}=9.14, p<.01\right.$, partial $\eta^{2}$ $=.29$ ) electrodes. Further simple effects tests revealed a significant effect of congruency in controls (lateral: $F_{(1,11)}=24.66, p<.001$, partial $\eta^{2}=.69$; midline: $F_{(1,11)}=12.43, p<.01$, partial $\eta^{2}=.53$ ), but not in amusics (lateral: $F_{(1,11)}=0.89, p=.37$, partial $\eta^{2}=.07$; midline: $F$ 
$(1,11)=0.34, p=.57$, partial $\left.\eta^{2}=.03\right)$. Furthermore, ANOVAs taking pitch distance as an additional within-subjects factor did not reveal any significant main effect or interaction related to interval distance, in either lateral or midline electrodes $(p s>.20)$.

\subsubsection{The impact of task on musical meaning processing}

To investigate the impact of task on musical meaning processing, five-factor and four-factor ANOVAs with task (implicit versus explicit) as an additional within-subjects factor were conducted for lateral and midline electrodes, respectively. No significant effect of task or interaction was observed for either lateral or midline electrodes $(p \mathrm{~s}>.14)$.

The abovementioned results demonstrated that incongruent targets did not elicit a larger N400 compared to congruent targets in either implicit or explicit tasks of iconic musical meaning processing for amusics. These EEG results are consistent with amusics' low accuracy in semantic congruency judgment in the behavioral task, indicating that amusics cannot associate direction of pitch change with iconic musical meaning. However, for controls, incongruent targets elicited a larger N400 than congruent targets over the anterior and central regions in the implicit task. Although the scalp distribution of this N400 effect also extended to the posterior electrodes in controls, no significant difference in the distribution of this N400 effect was observed between implicit and explicit tasks. The N400 effect observed for controls in both implicit and explicit tasks are consistent with what was reported for normal adults in previous studies (e.g., Daltrozzo \& Schön, 2009; Koelsch et al., 2004; Zhou et al., 2014; Zhou et al., 2015). Furthermore, our analysis did not find any effect of pitch distance on iconic musical meaning processing. This might be due to the fact that all 
of the melodies had a linear upward or downward trajectory, and the pitch distance between the first and last pitches was larger than one octave, which exceeded the pitch direction detection thresholds of amusic individuals (Jiang et al., 2013; Liu et al., 2010; Liu, Xu, et al., 2012).

\section{Experiment 2: Musical meaning embedded in natural music}

In Experiment 1, while controls showed a larger N400 effect in response to the incongruent condition relative to the congruent condition, amusics' brain activities did not differ significantly between the two conditions. This suggests that amusia is associated with reduced sensitivity to direction of pitch change at the cognitive level. Given that a variety of acoustic cues (such as pitch, tempo, rhythm, dynamics, and timbre) may contribute to iconic musical meaning processing (Zhou et al., 2015) and amusics are impaired primarily in the dimension of pitch perception, we predicted that amusics would be able to process meaning in natural musical excerpts where musical meaning can be interpreted using non-pitch-based cues, such as rhythm, dynamics, and timbre. Based on this hypothesis, Experiment 2 examined whether amusics would be able to activate the iconic representation of musical meaning in natural musical excerpts for both implicit and explicit tasks.

\subsection{Stimuli and procedure}

A total of 240 natural musical excerpts and 240 images were chosen from our previous stimuli (Zhou et al., 2014; Zhou et al., 2015). Images were semantically congruent or 
incongruent to musical excerpts (see Figure 5 and Supplemental Material for examples). As in Experiment 1, a pretest was conducted to ensure that the final selected 160 musical excerpts were semantically congruent or incongruent with 160 images. To rule out the influence of emotional congruency, which might be confounded with iconic musical meaning, another pretest was conducted to evaluate the emotional valence of each image (see Supplementary Material for details). No significant difference in emotional rating was found between the images in the selected pairs $(p \mathrm{~s}>.05)$, indicating that emotional valance was balanced between the congruent and incongruent conditions.

Insert Figure 5, about here.

The experimental procedure was identical to Experiment 1. Furthermore, an identification test was conducted after the EEG experiment in order to control for the familiarity effect of the musical excerpts (see Supplementary Material for details).

\subsection{Results and discussion}

\subsubsection{Implicit judgment task}

\subsubsection{Behavioral results}

Recognition accuracy of all probe stimuli (visual and auditory combined) was calculated. Participants provided correct responses to $88.54 \%(S D=8.66)$ of the probe stimuli, and no significant difference was found between the amusics and controls $\left(t_{(22)}=0.58, p=.57\right.$; 
amusics: $M=87.50 \%, S D=9.89$; controls: $M=89.58 \%, S D=7.52)$. This indicates that both groups attended to and were able to recognize the prime and target stimuli in the implicit task.

\subsubsection{Electrophysiological results}

Figure 6 shows the grand average waveforms elicited by target images following the congruent and incongruent priming of musical excerpts at 9 representative electrodes in the implicit task. The incongruent condition elicited a larger negativity with a peak latency of approximately $400 \mathrm{~ms}$ relative to the congruent condition for both groups. With such effects, the standard N400 time window of $300 \mathrm{~ms}$ to $500 \mathrm{~ms}$ was selected for statistical analysis.

Insert Figure 6, about here.

The analysis revealed no significant effect of group, for either lateral (main effect of group, $F_{(1,22)}=0.01, p=.94$, partial $\eta^{2}=.0002$ ) or midline (main effect of group, $F_{(1,22)}=$ $0.31, p=.58$, partial $\left.\eta^{2}=.014\right)$ electrodes. In addition, no significant interactions were observed between group and any of the other factors, for either lateral or midline electrodes $(p s>.48)$. Thus, the musical impairments that characterize congenital amusia do not seem to have a significant impact on the processing of iconic musical meaning in natural music.

The N400 amplitude was larger under the incongruent condition than under the congruent condition, for both lateral (main effect of congruency, $F_{(1,22)}=10.70, p<.01$, partial $\eta^{2}=.33$ ) and midline (main effect of congruency, $F_{(1,22)}=16.58, p<.01$, partial $\eta^{2}$ 
$=.43$ ) electrodes. In addition, we observed significant interactions between congruency and anteriority for both lateral and midline electrodes $\left(F_{(2,44)}=6.71, p<.05, \varepsilon=.56\right.$, partial $\eta^{2}$ $=.23$, and $F_{(2,44)}=9.32, p<.01, \varepsilon=.66$, partial $\left.\eta^{2}=.30\right)$. Results from further simple effects tests showed that incongruent targets elicited a larger N400 than congruent targets over the anterior (lateral: $F_{(1,23)}=14.47, p<.001$, partial $\eta^{2}=.39$; midline: $F_{(1,23)}=19.31, p<.001$, partial $\eta^{2}=.46$ ) and central (lateral: $F_{(1,23)}=9.93, p<.01$, partial $\eta^{2}=.30$; midline: $F_{(1,23)}=$ $18.06, p<.001$, partial $\left.\eta^{2}=.44\right)$ regions, but not over the posterior region (lateral: $F_{(1,23)}=$ $2.25, p=.15$, partial $\eta^{2}=.09$; midline: $F_{(1,23)}=1.74, p=.20$, partial $\left.\eta^{2}=.07\right)$.

\subsubsection{Explicit judgment task}

\subsubsection{Behavioral results}

No significant difference was found in the accuracy of semantic congruency judgment between the amusics and controls $\left(t_{(22)}=1.61, p=.12\right.$; amusics: $M=76.83 \%, S D=5.67$; controls: $M=81.00 \%, S D=6.95)$. Furthermore, no significant correlation was found between the global score of the MBEA tests and the accuracy of semantic congruency judgment, for amusics $\left(r_{(10)}=0.11, p=.73\right)$, controls $\left(r_{(10)}=0.02, p=.96\right)$, or both groups combined $\left(r_{(22)}=0.32, p=.13\right)$, indicating that musical abilities are not related to semantic congruency judgment.

\subsubsection{Electrophysiological results}

Figure 7 shows the grand average waveforms elicited by target images following the congruent and incongruent priming of musical excerpts at 9 representative electrodes in the 
explicit task. Similar to the implicit task, the incongruent condition elicited a larger negativity with a peak latency of approximately $400 \mathrm{~ms}$ relative to the congruent condition for both groups. With such effects, the standard N400 time window of $300 \mathrm{~ms}$ to $500 \mathrm{~ms}$ was selected for statistical analysis.

Insert Figure 7, about here.

The analysis revealed no significant effect of group, for either lateral (main effect of group, $F_{(1,22)}=0.47, p=.50$, partial $\eta^{2}=.02$ ) or midline (main effect of group, $F_{(1,22)}=0.86$, $p=.36$, partial $\left.\eta^{2}=.04\right)$ electrodes. In addition, no significant interactions were observed between group and the other factors for lateral or midline electrodes $(p s>.22)$. Thus, the musical impairments that characterize congenital amusia do not seem to have a significant impact on iconic musical meaning processing in natural music.

The N400 amplitude was larger under the incongruent condition than under the congruent condition, for both lateral (main effect of congruency, $F_{(1,22)}=16.77, p<.001$, partial $\eta^{2}=.43$ ) and midline electrodes (main effect of congruency, $F_{(1,22)}=11.65, p<.01$, partial $\eta^{2}=.35$ ). In addition, significant interactions between congruency and anteriority were observed for the lateral electrodes $\left(F_{(2,44)}=4.68, p<.05, \varepsilon=.56\right.$, partial $\left.\eta^{2}=.18\right)$. Results from further simple effects tests showed that, for the lateral electrodes, the incongruent target elicited a larger N400 than the congruent target over the anterior $\left(F_{(1,23)}=17.76, p<.001\right.$, partial $\left.\eta^{2}=.44\right)$ and central $\left(F_{(1,23)}=18.16, p<.001\right.$, partial $\left.\eta^{2}=.44\right)$ regions, but not over 
the posterior region $\left(F_{(1,23)}=2.80, p=.11\right.$, partial $\left.\eta^{2}=.11\right)$.

\subsubsection{The impact of task on musical meaning processing}

To investigate the impact of task on musical meaning processing, ANOVAs with task (implicit versus explicit) as an additional within-subjects factor were conducted across all participants. There was a significant interaction between task and anteriority (lateral: $F_{(2,44)}=$ 4.69, $p<.05, \varepsilon=.64$, partial $\eta^{2}=.18$; midline: $F_{(2,44)}=5.34, p<.05, \varepsilon=.67$, partial $\left.\eta^{2}=.20\right)$. Further simple effects tests showed that the explicit task elicited larger ERP amplitudes than the implicit task over the central (lateral: $F_{(1,23)}=18.08, p<.001$, partial $\eta^{2}=.44$; midline: $F$ $(1,23)=17.94, p<.001$, partial $\left.\eta^{2}=.44\right)$ and posterior (lateral: $F_{(1,23)}=7.40, p<.05$, partial $\eta^{2}$ $=.24$; midline: $F_{(1,23)}=7.97, p=.01$, partial $\left.\eta^{2}=.26\right)$ regions, but not over the anterior region (lateral: $F_{(1,23)}=0.002, p=.96$, partial $\eta^{2}<.001 ;$ midline: $F_{(1,23)}=0.08, p=.78$, partial $\left.\eta^{2}=.003\right)$. Although this indicated a slight centro-posterior weighting in explicit relative to implicit processing, the factor of task did not interact with other factors ( $p \mathrm{~s}>.26)$.

These results showed that, for both groups, incongruent targets elicited a larger N400 than congruent targets over the anterior and central regions in both implicit and explicit tasks. Moreover, the observed N400 effect in amusics and controls was consistent with their behavioral judgment of semantic congruency. Overall, these results indicated that, like controls, amusics were able to process iconic musical meaning in natural musical excerpts in both implicit and explicit tasks. Critically, there was no significant group difference in the amplitude or scalp distribution of the N400 effect, indicating that the processing of iconic meaning in natural musical excerpts was intact in amusics. These findings are consistent with 
previous results on preserved perception of musical emotion in amusia (Gosselin, Paquette, \& Peretz, 2015).

\section{General discussion}

The present study investigated the processing of iconic musical meaning in individuals with amusia, using implicit (recognition) and explicit (semantic congruency judgment) tasks in a cross-modal semantic priming paradigm. Unlike controls, amusics failed to elicit an N400 effect when iconic musical meaning was represented by direction of pitch change, in both implicit and explicit tasks. However, similar to controls, amusics demonstrated intact musical meaning processing for natural musical excerpts during both implicit and explicit judgment, as reflected by the elicitation of the N400 effects and their behavioral performance in the explicit task. Moreover, although explicit processing elicited larger ERP amplitudes than implicit processing over the centro-posterior regions, the nature of the tasks had no impact on iconic musical meaning processing for either amusics or controls. To the best of our knowledge, this is the first study to investigate the processing of musical meaning in amusia. Our results provide evidence in support of the "melodic contour deafness hypothesis" (Patel, 2008) by extending it to aspects of musical meaning perception.

A main contribution of the current study is to show abnormal brain activities underlying meaning processing elicited by direction of pitch change in amusia. Since we controlled for the changes of non-pitch-based acoustic cues in Experiment 1, the iconic musical meaning only resulted from the direction (upward or downward) of pitch changes. Although the 
deficits in discriminating pitch direction or melodic contour have been revealed in amusia (Foxton et al., 2004; Jiang et al., 2013; Liu et al., 2010; Liu, Xu, et al., 2012; Loui et al., 2008; Williamson et al., 2012), our results extended this finding by showing that amusics failed to process iconic musical meaning conveyed by direction of pitch change. Moreover, our correlation results indicated that participants' scores on the MBEA were not associated with their performance on semantic congruency judgment. This is consistent with previous studies suggesting that deficits in pitch perception are not related to short-term memory for tone sequences (Albouy et al., 2013), pitch expectations (Omigie et al., 2012), or musical syntax and tonality performance (Jiang et al., 2016), and implies that the amusic deficits may be pitch-relevant at both low and high levels of music processing. Thus, there may be a neural network that is specific to pitch processing in music. Although a recent study has begun to explore this issue (Norman-Haignere et al., 2016), more studies are needed to fully answer this question.

However, iconic meaning processing of natural music may be intact in amusia, as demonstrated by the N400 effects elicited by natural musical excerpts in both groups. This may be because amusics are capable of using non-pitch-based cues to decode iconic musical meaning. Indeed, our previous study showed that iconic meaning in natural musical excerpts was well predicted by non-pitch-based acoustic and structural cues, e.g., tempo, spectral flux, register, and intensity flux (Zhou et al., 2015). It has been reported that amusics are capable of decoding musical emotions expressed by multiple acoustic cues, although they showed reduced sensitivity to musical emotions when pitch-based musical mode was manipulated 
(Gosselin et al., 2015). Given that the expression of musical emotions belongs to indexical musical meaning, a dimension of extramusical meaning (Koelsch, 2012; Steinbeis \& Koelsch, 2008, 2011), it could be suggested that amusics have preserved ability to process extramusical meaning conveyed by natural music.

Although present in controls, the N400 effects in processing musical meaning through direction of pitch change were not observed for amusics in either the explicit or implicit task. This finding is contradictory to previous results of preserved sensitivity to musical structure (Tillmann et al., 2012; Zendel et al., 2015) and pitch expectations (Omigie et al., 2012) in implicit tasks in amusia. There are two possible reasons for this discrepancy.

One possible explanation may relate to the processing difference between musical structural regularity and musical meaning. The processing of musical structure and the expectation of melodic pitch is based primarily on musical structural regularity, which may be acquired through exposure to music (Rohrmeier \& Koelsch, 2012; Rohrmeier \& Rebuschat, 2012). On the contrary, musical meaning does not always represent a single concept, and the diversity of music makes implicit learning of musical meaning impossible (Patel, 2008). In our current study, iconic musical meaning is referred to as the association between music and extramusical iconic signs, and this kind of association is based on imitation. Alternatively, it might be the case that amusics fail to develop cross-modal associations between direction of pitch change and visual information necessary for semantic priming. Indeed, behavioral findings have demonstrated that even when auditory stimuli are concurrently presented with visual cues on a computer screen, amusics show worse 
performance than controls on melodic contour identification (Jiang et al., 2010) and pitch direction judgment (Liu, Jiang, Francart, Chan, \& Wong, in press), although they may benefit from using simultaneous visual stimuli to detect pitch changes (Albouy et al., 2015). An ERP study (Lu, Ho, Sun, Johnson, \& Thompson, 2016) confirms that amusics lack an enhanced $\mathrm{N} 2-\mathrm{P} 3$ response to incongruent audio-visual pairings in judging direction of pitch change in an implicit task. Given that the absence of N2-P3 complex is likely due to an unconscious neglect of audio-visual conflict (Lu et al., 2016), our current findings suggest that this neglect is likely to be also associated with reduced sensitivity to musical meaning represented by direction of pitch change in amusia.

The second explanation may come from the different stages of music processing. Using an implicit task, a previous ERP study reported an ERAN elicited by pitch deviants in amusics, suggesting that amusics show automatic brain response to musical structure (Zendel et al., 2015). ERAN is an electrophysiological index of syntactic processing in music, occurring in the early stage of syntactic processing (Koelsch, 2009). In contrast, the P600, an ERP component occurring at a relatively late stage of syntactic processing, was not found in amusics (Peretz, Brattico, Järvenpää, \& Tervaniemi, 2009; Zendel et al., 2015). Similarly, the processing of musical meaning in the present study, as reflected by the N400 effects, occurred at a temporally later stage of processing. It has also been suggested that top-down controlled mechanisms generally act more effectively when the target is primed with a long SOA (e.g., Anderson \& Holcomb, 1995; Lau, Phillips, \& Poeppel, 2008), although increasing the SOA of pitches per se (i.e., tone duration) might enhance the ability of pitch perception in 
amusics (Albouy, Cousineau, Caclin, Tillmann, \& Peretz, 2016). Given that we examined the N400 effect using a relatively long SOA (800 ms; see Supplementary Material for details), it was less likely for the brain to respond to the prime-target congruency automatically. Consequently, amusics exhibit impairment in processing musical meaning represented by direction of pitch change, even in the implicit task.

To conclude, our findings suggest that individuals with amusia are capable of extracting iconic musical meaning from natural musical excerpts, although they showed abnormal brain responses to the expression of musical meaning through direction of pitch change. Given that pitch is only one of the many perceptual cues contributing to music comprehension, it is understandable that deficits in pitch processing in amusia may only have minor impact on iconic musical meaning processing. From the perspective of meaning communication in speech, although changes in tone influence word meaning in tone languages, Mandarin-/Cantonese-speaking amusics could still speak and understand Mandarin/Cantonese (Liu et al., 2016; Nan, Sun, \& Peretz, 2010). Three possibilities may account for this phenomenon. First, pitch variations in language are coarse compared to those used in music, and this is true even for tone languages in which tones are differentiated by both contour and register (Liu et al., 2016; Liu, Jiang, et al., 2012). Second, speech communication relies not only on pitch variations, but also on other acoustic cues such as intensity, tempo, and timbre (Ilie \& Thompson, 2006). Third, segmental information and lexical constraints provided by sentence context may also aid speech intelligibility (Liu, Jiang, Wang, Xu, \& Patel, 2015). Indeed, even monotone sentences (with flattened F0) can be as 
intelligible as normal speech when heard in a quiet background for both normal and amusic individuals (Liu et al., 2015). Overall, our findings suggest that, although pitch is not an exclusive cue for communication in music and speech, it provides a unique perspective to investigate neural mechanisms of meaning processing in amusic as well as typically developing individuals. 


\section{Acknowledgments}

This work was supported by the National Natural Science Foundation of China (Grant No. 31470972 to C. J. and Grant No. 31500876 to L. Z.), and the European Research Council Starting Grant to F. L. and C. J. (CAASD, No. 678733). We wish to thank Prof. Stefan Koelsch and one anonymous reviewer for their insightful comments. 


\section{References}

Albouy, P., Cousineau, M., Caclin, A., Tillmann, B., \& Peretz, I. (2016). Impaired encoding of rapid pitch information underlies perception and memory deficits in congenital amusia. Scientific Reports, 6, 18861. doi: 10.1038/srep18861

Albouy, P., Lévêque, Y., Hyde, K. L., Bouchet, P., Tillmann, B., \& Caclin, A. (2015).

Boosting pitch encoding with audiovisual interactions in congenital amusia.

Neuropsychologia, 67, 111-120. doi: 10.1016/j.neuropsychologia.2014.12.006

Albouy, P., Mattout, J., Bouet, R., Maby, E., Sanchez, G., Aguera, P.-E., . . . Tillmann, B.

(2013). Impaired pitch perception and memory in congenital amusia: The deficit starts in the auditory cortex. Brain, 136(5), 1639-1661. doi: 10.1093/brain/awt082

Anderson, J. E., \& Holcomb, P. J. (1995). Auditory and visual semantic priming using different stimulus onset asynchronies: An event-related brain potential study. Psychophysiology, 32(2), 177-190. doi: 10.1111/j.1469-8986.1995.tb03310.x

Ayotte, J., Peretz, I., \& Hyde, K. (2002). Congenital amusia : A group study of adults afflicted with a music-specific disorder. Brain, 125(2), 238-251. doi:

10.1093/brain/awf028

Cook, N. (1987). The perception of large-scale tonal closure. Music Perception, 5(2), 197-205. doi: 10.2307/40285392 
Daltrozzo, J., \& Schön, D. (2009). Conceptual processing in music as revealed by N400 effects on words and musical targets. Journal of Cognitive Neuroscience, 21(10), 1882-1892. doi: 10.1162/jocn.2009.21113

Foxton, J. M., Dean, J. L., Gee, R., Peretz, I., \& Griffiths, T. D. (2004). Characterization of deficits in pitch perception underlying 'tone deafness'. Brain, 127(4), 801-810. doi: 10.1093/brain/awh105

Gosselin, N., Paquette, S., \& Peretz, I. (2015). Sensitivity to musical emotions in congenital amusia. Cortex, 71, 171-182. doi: 10.1016/j.cortex.2015.06.022

Henry, M. J., \& McAuley, J. D. (2013). Failure to apply signal detection theory to the Montreal Battery of Evaluation of Amusia may misdiagnose amusia. Music Perception, 30(5), 480-496. doi: 10.1525/mp.2013.30.5.480

Huron, D., \& Parncutt, R. (1993). An improved model of tonality perception incorporating pitch salience and echoic memory. Psychomusicology, 12(2), 154-171. doi:

$10.1037 / \mathrm{h} 0094110$

Hyde, K. L., \& Peretz, I. (2004). Brains that are out of tune but in time. Psychological Science, 15(5), 356-360. doi: 10.1111/j.0956-7976.2004.00683.x

Hyde, K. L., Zatorre, R. J., \& Peretz, I. (2011). Functional MRI evidence of an abnormal neural network for pitch processing in congenital amusia. Cerebral Cortex, 21(2), 292-299. doi: 10.1093/cercor/bhq094 
Ilie, G., \& Thompson, W. F. (2006). A comparison of acoustic cues in music and speech for three dimensions of affect. Music Perception, 23(4), 319-330. doi:

$10.1525 / \mathrm{mp} .2006 .23 .4 .319$

Jiang, C., Hamm, J. P., Lim, V. K., Kirk, I. J., \& Yang, Y. (2010). Processing melodic contour and speech intonation in congenital amusics with Mandarin Chinese. Neuropsychologia, 48(9), 2630-2639. doi: 10.1016/j.neuropsychologia.2010.05.009

Jiang, C., Hamm, J. P., Lim, V. K., Kirk, I. J., \& Yang, Y. (2011). Fine-grained pitch discrimination in congenital amusics with Mandarin Chinese. Music Perception, 28(5), 519-526. doi: 10.1525/mp.2011.28.5.519

Jiang, C., Lim, V. K., Wang, H., \& Hamm, J. P. (2013). Difficulties with pitch discrimination influences pitch memory performance: Evidence from congenital amusia. PloS ONE, 8(10), e79216. doi: 10.1371/journal.pone.0079216

Jiang, C., Liu, F., \& Thompson, W. F. (2016). Impaired explicit processing of musical syntax and tonality in a group of Mandarin-speaking congenital amusics. Music Perception, 33(4), 401-413.

Kamiyama, K. S., Abla, D., Iwanaga, K., \& Okanoya, K. (2013). Interaction between musical emotion and facial expression as measured by event-related potentials. Neuropsychologia, 51(3), 500-505. doi: 10.1016/j.neuropsychologia.2012.11.031

Koelsch, S. (2009). Music-syntactic processing and auditory memory: Similarities and differences between ERAN and MMN. Psychophysiology, 46(1), 179-190. doi: 10.1111/j.1469-8986.2008.00752.x 
Koelsch, S. (2011). Towards a neural basis of processing musical semantics. Physics of Life Reviews, 8(2), 89-105. doi: 10.1016/j.plrev.2011.04.004

Koelsch, S. (2012). Brain and music. Oxford, UK: Wiley-Blackwell.

Koelsch, S., Kasper, E., Sammler, D., Schulze, K., Gunter, T., \& Friederici, A. D. (2004).

Music, language and meaning: Brain signatures of semantic processing. Nature Neuroscience, 7(3), 302-307. doi: 10.1038/nn1197

Lau, E. F., Phillips, C., \& Poeppel, D. (2008). A cortical network for semantics:(de) constructing the N400. Nature Reviews Neuroscience, 9(12), 920-933. doi: $10.1038 / \mathrm{nrn} 2532$

Liu, F., Chan, A. H. D., Ciocca, V., Roquet, C., Peretz, I., \& Wong, P. C. M. (2016). Pitch perception and production in congenital amusia: Evidence from Cantonese speakers. The Journal of the Acoustical Society of America, 140(1), 563-575. doi: 10.1121/1.4955182

Liu, F., Jiang, C., Francart, T., Chan, A. H., \& Wong, P. C. M. (in press). Perceptual learning of pitch direction in congenital amusia: Evidence from chinese speakers. Music Perception.

Liu, F., Jiang, C., Thompson, W. F., Xu, Y., Yang, Y., \& Stewart, L. (2012). The mechanism of speech processing in congenital amusia: Evidence from Mandarin speakers. PLoS ONE, 7(2), e30374. doi: 10.1371/journal.pone.0030374

Liu, F., Jiang, C., Wang, B., Xu, Y., \& Patel, A. D. (2015). A music perception disorder (congenital amusia) influences speech comprehension. Neuropsychologia, 66, 111-118. doi: 10.1016/j.neuropsychologia.2014.11.001 
Liu, F., Patel, A. D., Fourcin, A., \& Stewart, L. (2010). Intonation processing in congenital amusia: Discrimination, identification and imitation. Brain, 133(6), 1682-1693. doi: 10.1093/brain/awq089

Liu, F., Xu, Y., Patel, A. D., Francart, T., \& Jiang, C. (2012). Differential recognition of pitch patterns in discrete and gliding stimuli in congenital amusia: Evidence from Mandarin speakers. Brain and Cognition, 79(3), 209-215. doi: 10.1016/j.bandc.2012.03.008

Loui, P., Alsop, D., \& Schlaug, G. (2009). Tone deafness: A new disconnection syndrome? The Journal of Neuroscience, 29(33), 10215-10220. doi:

\subsection{3/JNEUROSCI.1701-09.2009}

Loui, P., Guenther, F. H., Mathys, C., \& Schlaug, G. (2008). Action-perception mismatch in tone-deafness. Current Biology, 18(8), R331-R332. doi: 10.1016/j.cub.2008.02.045

Lu, X., Ho, H. T., Sun, Y., Johnson, B. W., \& Thompson, W. F. (2016). The influence of visual information on auditory processing in individuals with congenital amusia: An ERP study. NeuroImage, 135, 142-151. doi: 10.1016/j.neuroimage.2016.04.043

Meltzoff, A. N., \& Prinz, W. (2002). The imitative mind: Development, evolution and brain bases. Cambridge: Cambridge University Press.

Nagy, E. (2006). From imitation to conversation: The first dialogues with human neonates. Infant and Child Development, 15(3), 223-232. doi: 10.1002/icd.460

Nan, Y., Sun, Y., \& Peretz, I. (2010). Congenital amusia in speakers of a tone language: association with lexical tone agnosia. Brain, 133(9), 2635-2642. 
Norman-Haignere, S. V., Albouy, P., Caclin, A., McDermott, J. H., Kanwisher, N. G., \& Tillmann, B. (2016). Pitch-responsive cortical regions in congenital amusia. The Journal of Neuroscience, 36(10), 2986-2994. doi: 10.1523/JNEUROSCI.2705-15.2016

Oldfield, R. C. (1971). The assessment and analysis of handedness: The Edinburgh inventory. Neuropsychologia, 9(1), 97-113. doi: 10.1016/0028-3932(71)90067-4

Omigie, D., Pearce, M. T., \& Stewart, L. (2012). Tracking of pitch probabilities in congenital amusia. Neuropsychologia, 50(7), 1483-1493. doi:

10.1016/j.neuropsychologia.2012.02.034

Painter, J. G., \& Koelsch, S. (2011). Can out-of-context musical sounds convey meaning? An ERP study on the processing of meaning in music. Psychophysiology, 48(5), 645-655. doi: 10.1111/j.1469-8986.2010.01134.x

Patel, A. D. (2008). Music, language, and the brain. New York: Oxford University Press.

Peretz, I., Ayotte, J., Zatorre, R. J., Mehler, J., Ahad, P., Penhune, V. B., \& Jutras, B. (2002). Congenital amusia: A disorder of fine-grained pitch discrimination. Neuron, 33(2), 185-191. doi: 10.1016/S0896-6273(01)00580-3

Peretz, I., Brattico, E., Järvenpää, M., \& Tervaniemi, M. (2009). The amusic brain: In tune, out of key, and unaware. Brain, 132(5), 1277-1286. doi: 10.1093/brain/awp055

Pfeifer, J., \& Hamann, S. (2015). Revising the diagnosis of congenital amusia with the Montreal Battery of Evaluation of Amusia. Frontiers in Human Neuroscience, 9, 161. doi: 10.3389/fnhum.2015.00161 
Rohrmeier, M., \& Koelsch, S. (2012). Predictive information processing in music cognition. A critical review. International Journal of Psychophysiology, 83(2), 164-175. doi: 10.1016/j.ijpsycho.2011.12.010

Rohrmeier, M., \& Rebuschat, P. (2012). Implicit learning and acquisition of music. Topics in Cognitive Science, 4(4), 525-553. doi: 10.1111/j.1756-8765.2012.01223.x

Steinbeis, N., \& Koelsch, S. (2008). Comparing the processing of music and language meaning using EEG and fMRI provides evidence for similar and distinct neural representations. PLoS ONE, 3(5), e2226. doi: 10.1371/journal.pone.0002226

Steinbeis, N., \& Koelsch, S. (2011). Affective priming effects of musical sounds on the processing of word meaning. Journal of Cognitive Neuroscience, 23(3), 604-621. doi: 10.1162/jocn.2009.21383

Tillmann, B., Gosselin, N., Bigand, E., \& Peretz, I. (2012). Priming paradigm reveals harmonic structure processing in congenital amusia. Cortex, 48(8), 1073-1078. doi: 10.1016/j.cortex.2012.01.001

Tillmann, B., Lévêque, Y., Fornoni, L., Albouy, P., \& Caclin, A. (2016). Impaired short-term memory for pitch in congenital amusia. Brain Research, 1640, 251-263. doi: 10.1016/j.brainres.2015.10.035

Tillmann, B., Lalitte, P., Albouy, P., Caclin, A., \& Bigand, E. (2016). Discrimination of tonal and atonal music in congenital amusia: The advantage of implicit tasks. Neuropsychologia, 85, 10-18. doi: 10.1016/j.neuropsychologia.2016.02.027 
van Zuijen, T. L., Simoens, V. L., Paavilainen, P., Näätänen, R., \& Tervaniemi, M. (2006). Implicit, intuitive, and explicit knowledge of abstract regularities in a sound sequence: an event-related brain potential study. Journal of Cognitive Neuroscience, 18(8), 1292-1303. doi: 10.1162/jocn.2006.18.8.1292

Williamson, V. J., Liu, F., Peryer, G., Grierson, M., \& Stewart, L. (2012). Perception and action de-coupling in congenital amusia: Sensitivity to task demands. Neuropsychologia, 50(1), 172-180. doi: 10.1016/j.neuropsychologia.2011.11.015

Zendel, B. R., Lagrois, M.-É., Robitaille, N., \& Peretz, I. (2015). Attending to pitch information inhibits processing of pitch information: The curious case of amusia. The Journal of Neuroscience, 35(9), 3815-3824. doi: 10.1523/JNEUROSCI.3766-14.2015

Zhou, L., Jiang, C., Delogu, F., \& Yang, Y. (2014). Spatial conceptual associations between music and pictures as revealed by N400 effect. Psychophysiology, 51(6), 520-528. doi: 10.1111/psyp.12195

Zhou, L., Jiang, C., Wu, Y., \& Yang, Y. (2015). Conveying the concept of movement in music: An event-related brain potential study. Neuropsychologia, 77, 128-136. doi: 10.1016/j.neuropsychologia.2015.07.029 


\section{Table}

Table 1. Participants' characteristics and mean scores of the MBEA for each group. Standard deviation values are shown in parentheses.

\begin{tabular}{lclc}
\hline & $\begin{array}{l}\text { Amusic } \\
(\boldsymbol{n}=\mathbf{1 2})\end{array}$ & $\begin{array}{l}\text { Control } \\
(\boldsymbol{n}=\mathbf{1 2})\end{array}$ & $\boldsymbol{t}$-test \\
\hline Mean age $(S D)$ & $23(1.7)$ & $23(1.4)$ & n.s. \\
\hline Sex & $9 \mathrm{~F}, 3 \mathrm{M}$ & $9 \mathrm{~F}, 3 \mathrm{M}$ & n.s. \\
\hline Years education $(S D)$ & $17(1.4)$ & $18(0.9)$ & $p<.001$ \\
\hline Melodic score of MBEA $(S D)$ & $17(3.2)$ & $27(1.2)$ & $p<.001$ \\
\hline Global score of MBEA $(S D)$ & $18(3.6)$ & $28(0.7)$ & $p<.001$ \\
\hline d' of MBEA $(S D)$ & $0.91(0.25)$ & $2.87(0.32)$ & \\
\hline
\end{tabular}

Note: $\mathrm{F}=$ female; $\mathrm{M}=$ male. The "Melodic score of MBEA" refers to the mean score of the three pitch-based subtests. 


\section{Figure captions}

Figure 1. Electrode layout on the scalp. The electrodes selected for statistical analysis were grouped into six regions for the lateral electrodes: left and right anterior, left and right central, and left and right posterior. For the midline electrodes, three regions were defined: midline-anterior, midline-central, and midline-posterior.

Figure 2. Design of the cross-modal semantic priming paradigm in Experiment 1. Musical excerpts characterized by direction (upward or downward) of pitch change with a tempo of 70 beats per minute were used as primes, and images depicting upward or downward movements were used as targets. According to the rating results of semantic congruency, target images are congruent or incongruent to the prime music. SOA: stimulus onset asynchrony.

Figure 3. Grand mean ERPs recorded during the implicit (recognition) task in Experiment 1. (A) Grand mean ERP waveforms elicited by congruent and incongruent target images at 9 electrode sites for amusics and controls, respectively. Gray-shaded areas indicate the time window used for statistical analysis. (B) Incongruent-minus-congruent difference waves for amusics (solid line) and controls (dotted line). (C) Scalp distribution of the incongruent-minus-congruent difference waves in the $300-500$ ms latency range for amusics and controls, respectively.

Figure 4. Grand mean ERPs recorded during the explicit (semantic congruency judgment) task in Experiment 1. (A) Grand mean ERP waveforms elicited by congruent and incongruent 
target images at 9 electrode sites for amusics and controls, respectively. Gray-shaded areas indicate the time window used for statistical analysis. (B) Incongruent-minus-congruent difference waves for amusics (solid line) and controls (dotted line). (C) Scalp distribution of the incongruent-minus-congruent difference waves in the $300-500 \mathrm{~ms}$ latency range for amusics and controls, respectively.

Figure 5. Design of the cross-modal semantic priming paradigm in Experiment 2. Musical excerpts selected from natural music with a duration of $3 \mathrm{~s}$ were used as primes, and images were used as targets. According to the rating results of semantic congruency, target images are congruent or incongruent to the prime music.

Figure 6. Grand mean ERPs recorded during the implicit (recognition) task in Experiment 2.

(A) Grand mean ERP waveforms elicited by congruent and incongruent target images at 9 electrode sites for amusics and controls, respectively. Gray-shaded areas indicate the time window used for statistical analysis. (B) Incongruent-minus-congruent difference waves for amusics (solid line) and controls (dotted line). (C) Scalp distribution of the incongruent-minus-congruent difference waves in the $300-500$ ms latency range for amusics and controls, respectively.

Figure 7. Grand mean ERPs during the explicit (semantic congruency judgment) task in Experiment 2. (A) Grand mean ERP waveforms elicited by congruent and incongruent target images at 9 electrode sites for amusics and controls, respectively. Gray-shaded areas indicate the time window used for statistical analysis. (B) Incongruent-minus-congruent difference waves for amusics (solid line) and controls (dotted line). (C) Scalp distribution of the 
incongruent-minus-congruent difference waves in the 300 - 500 ms latency range for amusics and controls, respectively. 
Figure 1

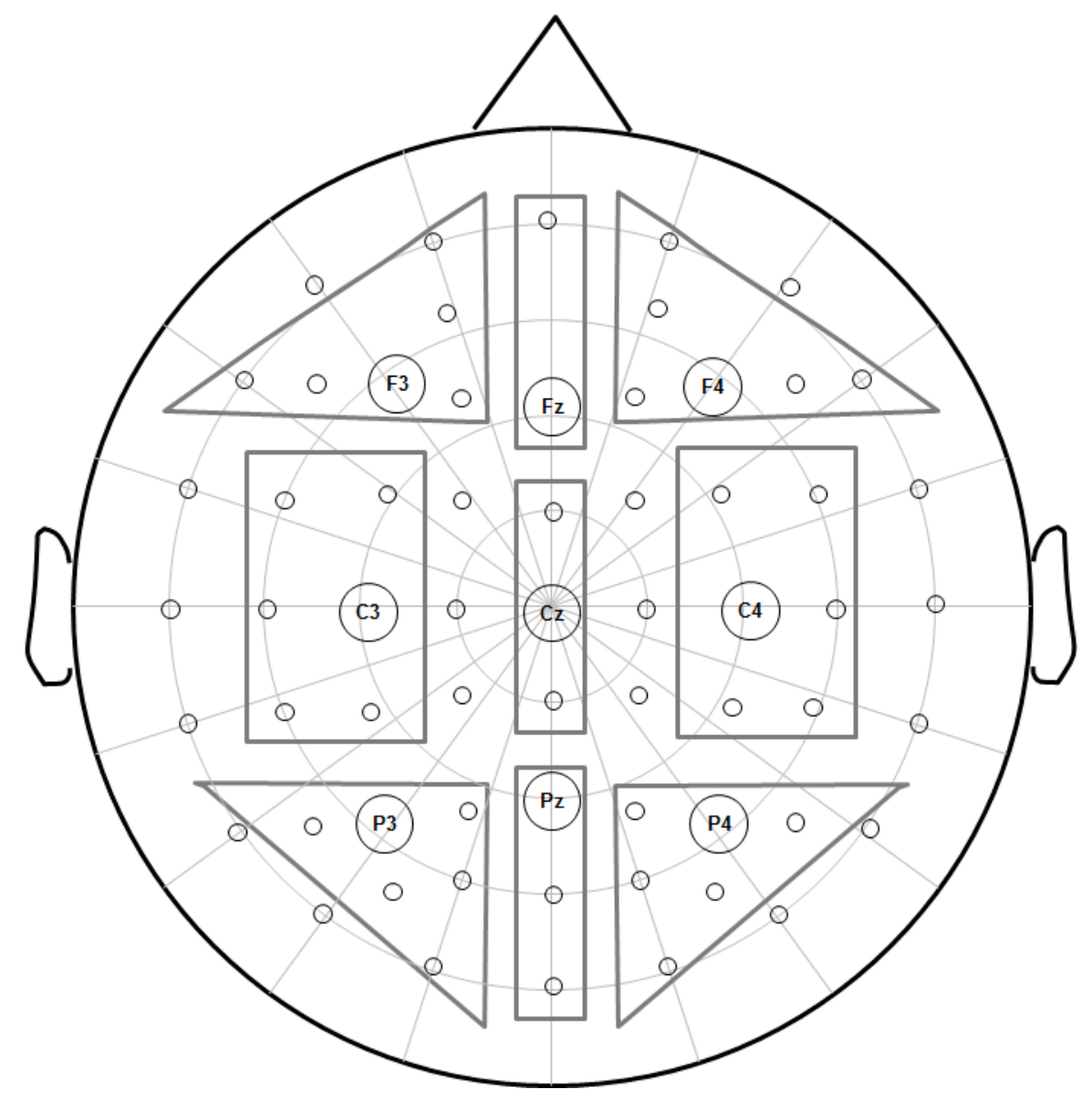


Figure 2

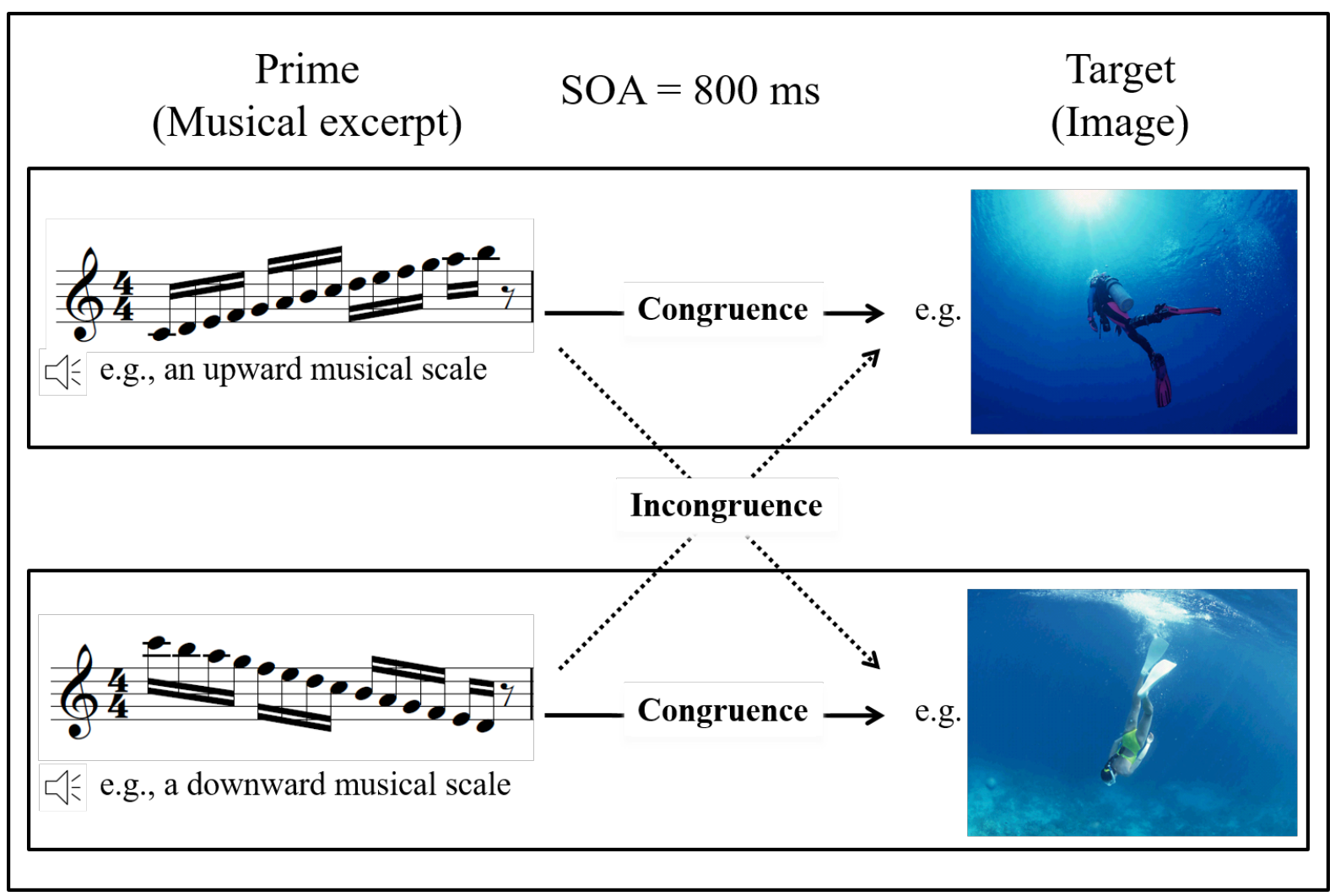


Figure 3

(A)




Incongruent



(B)

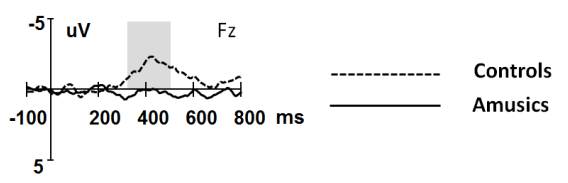

(C)




Figure 4

(A)

(2)

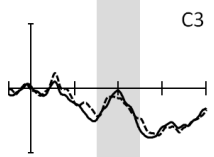

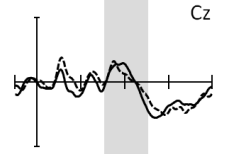
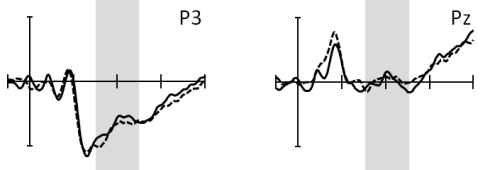
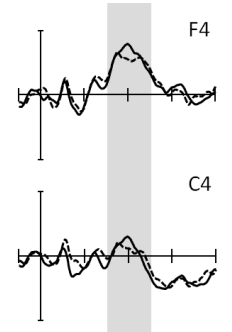



P4
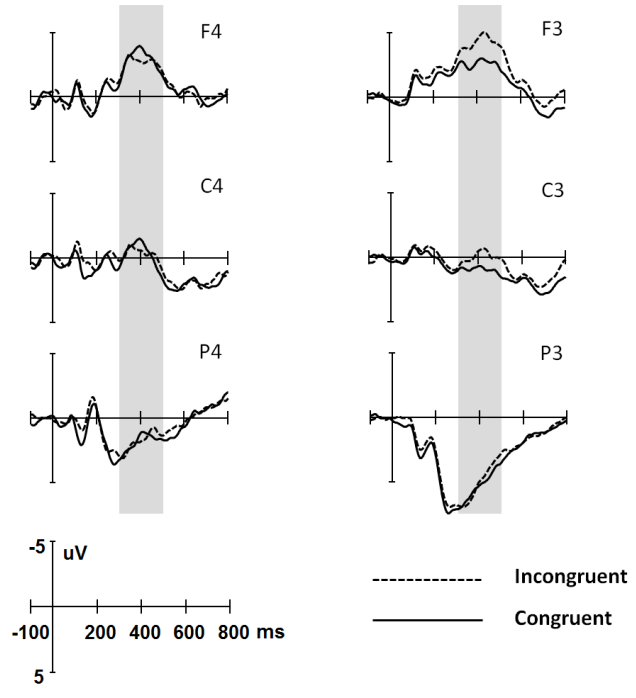

(C)



(B)

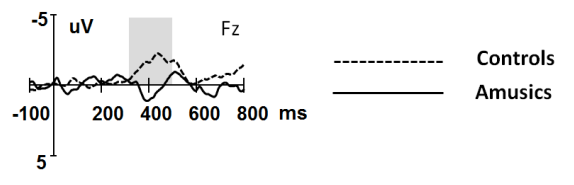

P3
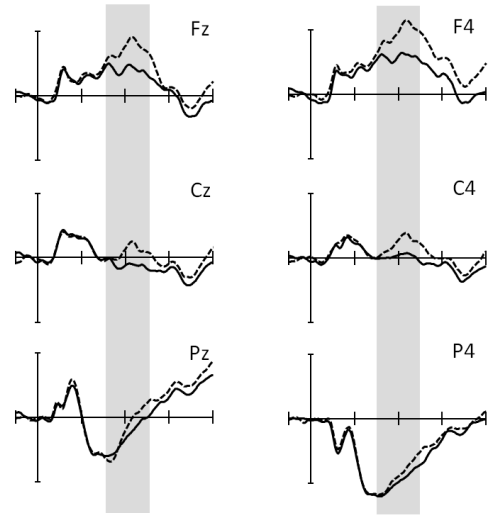

$\mathrm{Cz}$

P4
$300-500 \mathrm{~ms}$ 
Figure 5

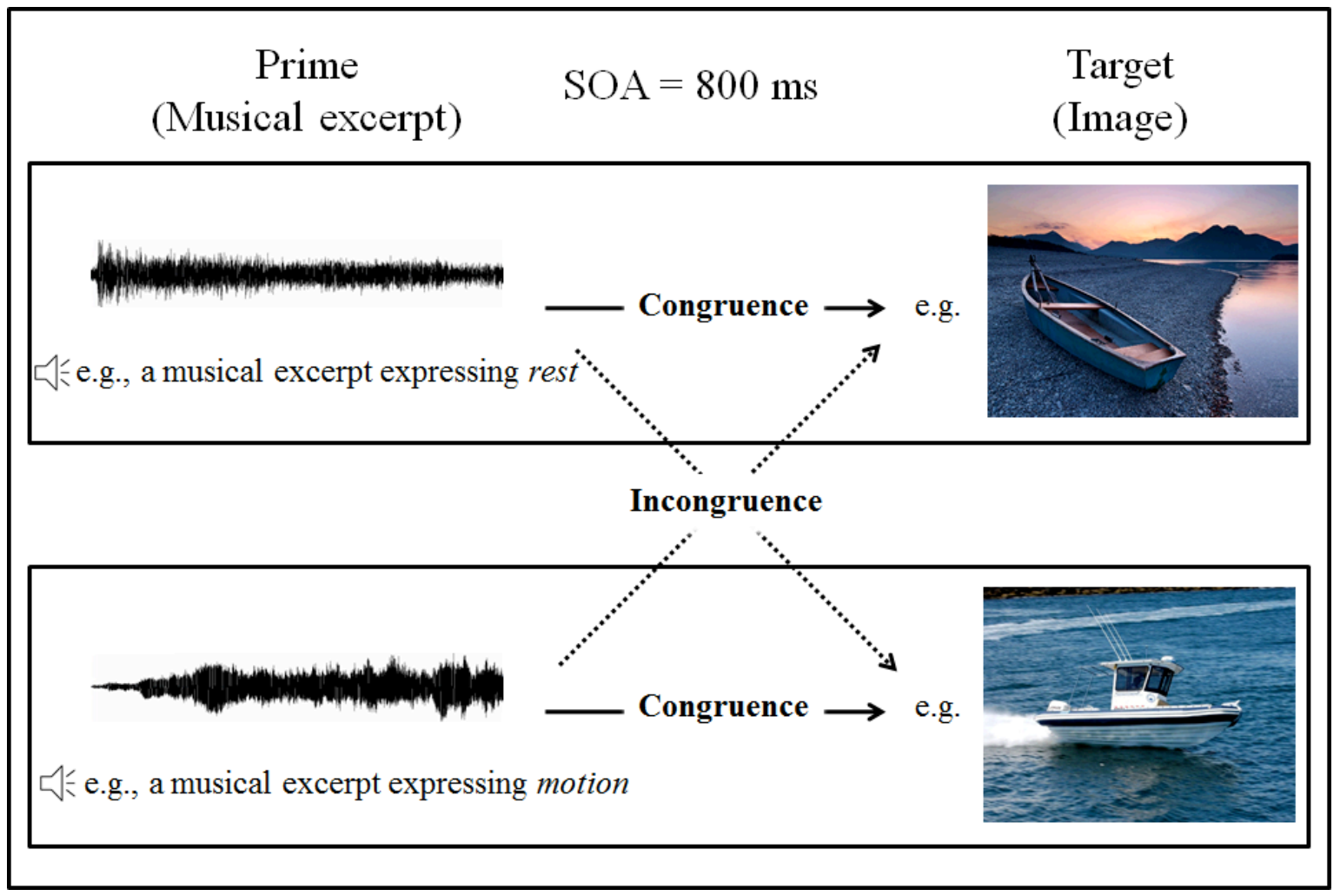




\section{Figure 6}

(A)


(B)

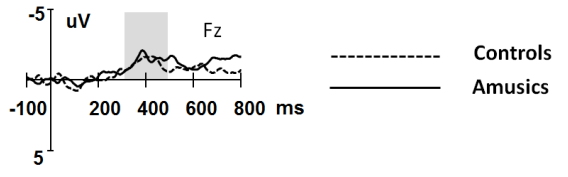

\section{Controls}
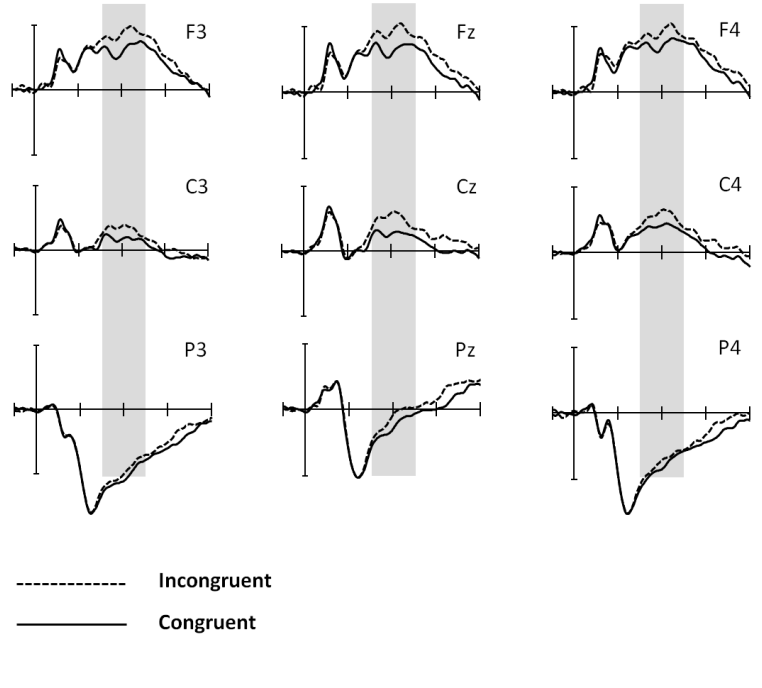

(C)


$300-500 \mathrm{~ms}$

$300-500 \mathrm{~ms}$ 
Figure 7

(A)
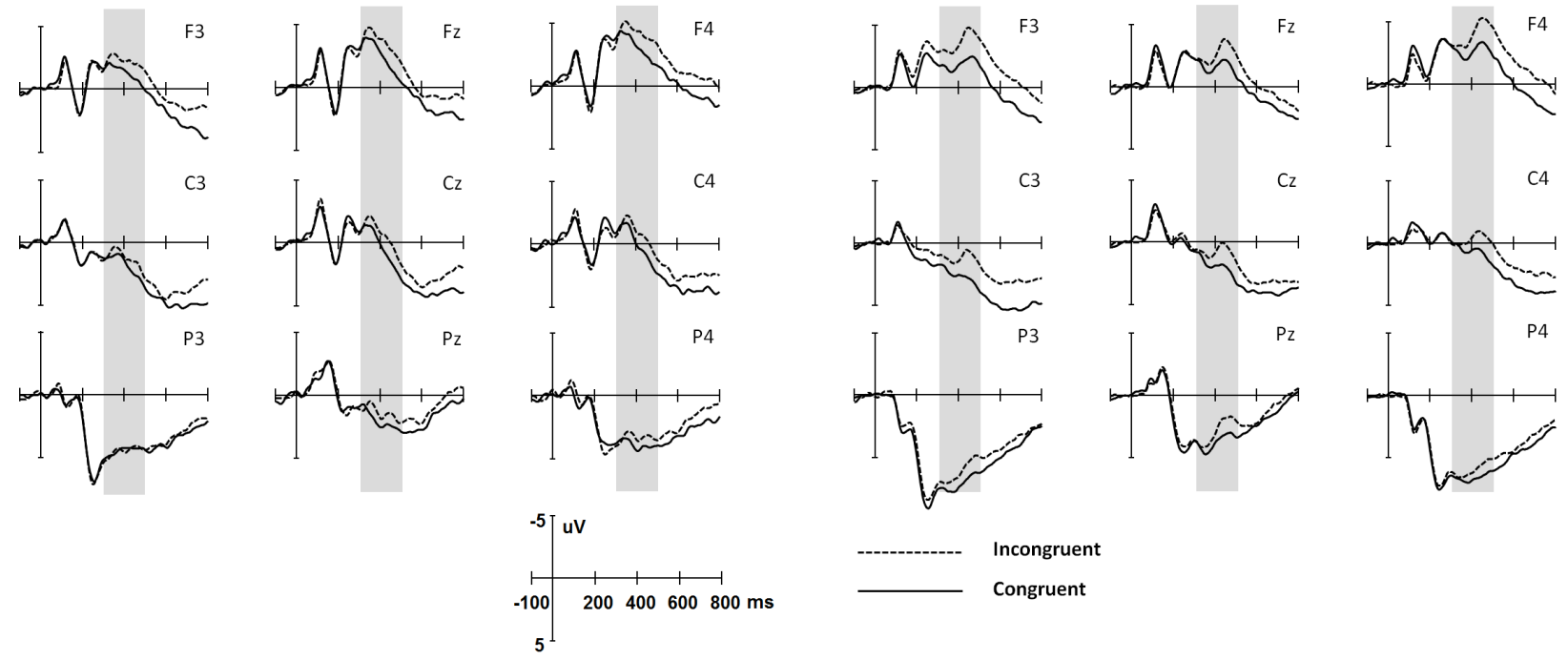

(B)
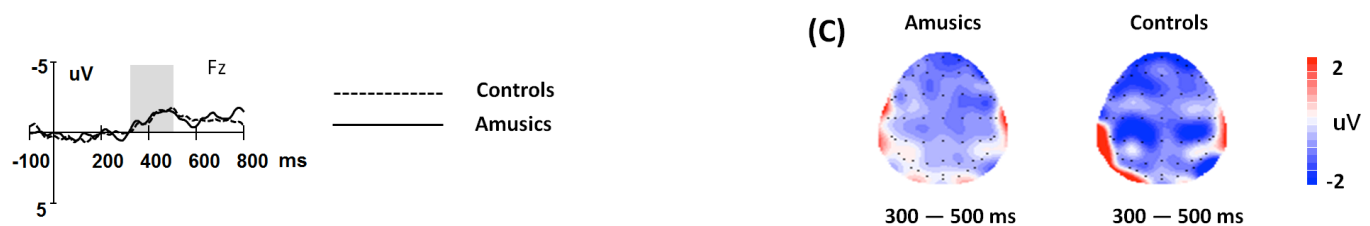